Trabajos Originales

\title{
Uso de pesarios en mujeres menores de 65 años con diagnóstico de prolapso de órganos pelvianos: estudio de factibilidad
}

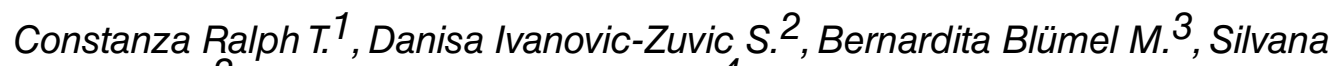 \\ Gonzalez L. ${ }^{3}$, Javier Pizarro-Berdichevsky. ${ }^{4}$
}

${ }^{1}$ Complejo Asistencial Hospital Dr. Sotero del Rio / Pontificia Universidad Católica de Chile, ${ }^{2}$ Escuela de Medicina, Pontificia Universidad Católica de Chile. ${ }^{3}$ Complejo Asistencial Hospital Dr. Sotero del Rio. ${ }^{4}$ Complejo Asistencial Hospital Dr. Sotero del Rio / Pontificia Universidad Católica de Chile, Glickman Urologic and Kidney Institute - Cleveland Clinic, Ohio, EEUU.

\section{RESUMEN}

Antecedentes: El prolapso de los órganos pelvianos (POP) es una enfermedad prevalente. Los pesarios vaginales son una opción de tratamiento. Algunos especialistas prefieren la cirugía como el tratamiento de elección. Objetivo: Describir la respuesta subjetiva y complicaciones asociadas al uso de pesarios en pacientes $\leq 65$ años con POP en el Hospital Dr. Sotero del Río. Métodos: Estudio retrospectivo de pacientes con POP sintomático $\leq 65$ años con indicación de pesario, entre julio de 2009 y noviembre de 2012. Para la evaluación de los resultados subjetivos del uso de pesarios, se utilizó la escala PGI-I. Resultados: Cuarenta y nueve pacientes cumplieron los criterios de inclusión. En 39 pacientes el fitting fue exitoso. Veinticinco de las 39 pacientes, fueron capaces de acceder y usar el pesario, de las cuales 6 abandonaron el tratamiento. La mediana de la edad fue 62,5 años. Un $48 \%$ eran sexualmente activas. La mediana de seguimiento fue 9 semanas. Según POP-Q un $60 \%$ se encontraba en etapa III. El tipo de pesario más utilizado fue el Gellhorn. Trece pacientes presentaron efectos adversos, siendo lo más frecuente el flujo vaginal (40\%). La respuesta subjetiva de mejoría estuvo disponible en $15(64 \%)$ de 25 pacientes. Un $88 \%$ notaron mejoría subjetiva importante. Conclusión: El uso de pesarios vaginales en pacientes menores de 65 años con POP es factible, asociado a mejoría subjetiva de los síntomas, con baja tasa de complicaciones. Son necesarios estudios prospectivos de mayor tamaño muestral para evaluar este subgrupo.

\section{PALABRAS CLAVE: Prolapso de los órganos pelvianos, pesario, manejo conservador, mujeres $<65$ años}

\section{SUMMARY}

Background: Pelvic organ prolapse (POP) is a common disease. Vaginal pessaries are a treatment option. Some specialists prefer surgery as the treatment of choice. Objective: To describe the subjective response and complications associated with the use of pessaries in patients $\leq 65$ years old with POP in Dr. Sotero del Rio Hospital. Methods: Retrospective study of patients with symptomatic POP $\leq 65$ and use pessary between July 2009 and November 2012. And assessment of the subjective results of using the PGI-I pessaries scale was used. Results: 49 patients met the inclusion criteria. In 39 patients the fitting was successful. 25 of 39 patients were able to access and use the pessary. 6 patients discontinued treatment. The median age was 62.5 years. $48 \%$ were sexually active. Median follow-up was 9 weeks. According POP- Q $60 \%$ were in 
stage III. The type of pessary was the most used Gellhorn. 13 patients had adverse effects. The subjective response improvement was available in $15(64 \%)$ of 25 patients. $88 \%$ noted significant subjective improvement. Conclusion: The use of vaginal pessaries in patients under 65 with POP is feasible, associated with subjective improvement in symptoms, with low complication rate. Prospective studies are needed with larger sample sizes to evaluate this subgroup.

\section{KEY WORDS: Pelvic organ prolapse, pessary, conservative management, women $<65$ years}

\section{INTRODUCCIÓN}

El prolapso de los órganos pelvianos (POP) tiene una prevalencia de vida del $30-50 \%$ (1), afectando severamente la calidad de vida de las pacientes $(2,3)$. Flores y cols, reportaron que en pacientes con POP presenta disminución del deseo sexual, dispareunia y disminución de la intensidad del orgasmo (4).

Los pesarios vaginales son una opción de tratamiento (5). Son dispositivos que se insertan en la vagina para reducir el POP. De esta forma, pueden aliviar los síntomas asociados $(5,6)$. Es un tratamiento mínimamente invasivo, de bajo costo, seguro y efectivo $(6,8)$. Ha sido usado desde comienzos de la historia, siendo ya descrito por Hipócrates en el año 400 a.C., constituyendo hasta el siglo XIX la principal modalidad de tratamiento para el POP $(6,7,8)$.

Un alto porcentaje de los especialistas y subespecialistas consideran la cirugía como el tratamiento de elección, relegando los pesarios sólo a pacientes con contraindicación quirúrgica o deseo de embarazo (9).

Kapoor y cols, demostraron que dos tercios de las pacientes optaban por el uso de pesario por sobre la cirugía como manejo inicial, cuando éste es ofrecido como alternativa (10). Un estudio controlado randomizado (11) que comparó el uso de pesario versus cirugía reportó similares resultados entre estas dos terapias. Sin embargo, hubo un $32 \%$ de pérdida de seguimiento programado en 1 año, dificultando la correcta interpretación de los resultados.

Diferentes estudios concluyen que un factor independiente de éxito para el uso de pesario es tener más de 65 años (12). Sin embargo, no existen en la literatura estudios que muestren los resultados en mujeres menores de 65 años. Sólo dos estudios hacen referencia al beneficio del uso de pesarios en mujeres sexualmente activas $(13,14)$. Bugge y cols reportaron en base a una encuesta realizada a los miembros de la International Urogy- necology Association que la impresión de los encuestados ( $98 \%$ médicos) fue que el subgrupo con mayor adherencia al uso de pesarios y que a su vez más lo solicitaba, eran las mujeres de mayor edad o las que tuviesen alguna contraindicación para operarse (15). Es destacable la mención a la falta de indicaciones para el uso de pesarios en mujeres jóvenes. Pese a lo anterior, las verdaderas preferencias de las pacientes siguen siendo desconocidas. Es posible que la información entregada por el personal de salud pudiera estar sesgada a favor de la cirugía en pacientes menores de 65 años.

El objetivo de este estudio es describir la respuesta subjetiva y complicaciones asociadas al uso de pesarios en pacientes $\leq 65$ años con POP a mediano plazo de seguimiento en el Hospital Dr. Sotero del Río (HSR). Nuestra hipótesis es que el uso de pesarios vaginales en pacientes menores de 65 años es factible, asociado a mejoría subjetiva de los síntomas de las pacientes, con baja tasa de complicaciones.

\section{PACIENTES Y MÉTODOS}

Se realizó un estudio retrospectivo de cohorte de pacientes con POP sintomático, $\leq 65$ años y uso de pesario entre julio de 2009 y noviembre de 2012. Los criterios de inclusión fueron: mujeres $\leq 65$ años con POP sintomático, $\geq$ etapa II, a quienes se les realizó una sesión de prueba (fitting) del pesario durante el período de estudio. Las características demográficas y clínicas de las pacientes se obtuvieron de la base de datos uroginecológica del HSR.

Para evaluar los resultados subjetivos del uso de pesarios, se utilizó la escala PGI-I (Patient Global Improvement Index) (16). Esta encuesta evalúa la impresión subjetiva de mejoría en base a una pregunta única ("¿Siente que en relación al período previo al uso del pesario usted se encuentra excelente, mucho mejor, un poco mejor, igual, un poco peor, mucho peor o pésimo?). Luego, la muestra se dividió entre pacientes que refirieron mejorar ("excelente, mucho mejor, un poco mejor") y las que 
no lo hicieron ("igual, un poco peor, mucho peor o pésimo"). Fitting exitoso, se definió como la sensación confortable del pesario y la no expulsión del dispositivo, luego de 40-60 minutos de la inserción manteniendo actividades habituales (caminar, subir y bajar escaleras, orinar y/o defecar) (11).

La metodología del trabajo, las definiciones y unidades de medida fueron consideradas según los estándares recomendados en conjunto entre la International Continence Society y la International Urogynecological Association, exceptuando donde explícitamente se expresa lo contrario. El protocolo de este estudio fue revisado y aprobado por el comité de ética HSR.

\section{RESULTADOS}

En el periodo del estudio, se evaluaron 179 pacientes con POP que asistieron a evaluación para fitting de pesario. Un 27\% (49 pacientes) cumplieron los criterios de inclusión. En 39 pacientes $(79,5 \%)$ el fitting fue exitoso. De este total, 25 de 39 $(66 \%)$ fueron capaces de acceder y usar el pesario como tratamiento. El $34 \%$ restante no fue capaz de adquirirlo por limitaciones económicas.

Las características demográficas son descritas en la Tabla I. La mediana de la edad fue 62,5 años. Un $48 \%$ eran sexualmente activas. La mediana de seguimiento fue 9 semanas.

Respecto a la etapificación, según POP-Q, un $60 \%$ se encontraba en etapa III, $20 \%$ etapa IV, $16 \%$ etapa II y $4 \%$ etapa I. Al momento del fitting, el tipo de pesario más utilizado fue el Gellhorn, seguido por el anillo con membrana (Figura 1 y 2). Del total de 25 pacientes que usaron pesario, 13 presentaron efectos adversos (Tabla II), lo más frecuente

\section{Tabla I \\ CARACTERÍSTICAS DEMOGRÁFICAS DE LAS PACIENTES $\leq 65$ AÑOS USUARIAS DE PESARIO}

\begin{tabular}{lr}
\hline Variable & \multicolumn{1}{c}{ Medida } \\
\hline Seguimiento (semanas) & $9(4,5-90)$ \\
Edad (años) & $62,5(49-65)$ \\
Paridad & $3(2-7)$ \\
Post Menopausia & $84 \%(21 / 25)$ \\
Actividad sexual & $48 \%(12 / 25)$ \\
Historia de cirugía por POP & $1(4 \%)$ \\
Peso máximo de recién nacido $(\mathrm{g})$ & $3930 \pm 514$ \\
IMC (kg/m2) & $29,1 \pm 2,9$ \\
\hline
\end{tabular}

Los resultados son presentados como mediana (rango intercuartil) o promedio $\pm \mathrm{DE}$. fue el flujo vaginal (40\%). Todas estas complicaciones fueron manejadas sin dificultad y las pacientes quedaron libres de secuelas. Sin embargo, 6 pacientes (23\%) abandonaron el tratamiento por molestias como flujo vaginal, sangrado genital o sensación de expulsión del pesario. La respuesta subjetiva de mejoría evaluada con el PGI-I estuvo disponible en 15 (64\%) de 25 pacientes. Un 88\% (14 pacientes) notaron mejoría subjetiva con respecto al periodo previo al uso del pesario (Tabla III).

\section{DISCUSIÓN}

El uso de pesarios ha demostrado ser efectivo en mejorar los síntomas asociados al POP, como el bulto y síntomas asociados a irritación vesical. A su vez, mejoran la percepción de la imagen corporal, calidad de vida, y satisfacción sexual $(6,8)$. Específicamente, el equipo de Lamers y cols, reportaron una tasa de $70-92 \%$ de mejoría en la satisfacción con el uso de pesarios a mediano plazo (3 meses) (6). En nuestro estudio, la mejoría subjetiva fue alta $(88 \%)$, comparable con otros estudios y con tratamientos quirúrgicos para el POP. Nuestro estudio tiene la debilidad de tener un tamaño muestral pequeño y no contar con el resultado de PGI-I en todas las pacientes. Considerando un análisis de peor escenario de la respuesta subjetiva de nuestros resultados, es decir que en las pacientes en que PGI-I no estuvo disponible no hubo mejoría, esta sigue siendo suficientemente alta (56\%). Este análisis, aunque ficticio, es útil para considerar a los pesarios como parte de las opciones terapéuticas en mujeres $\leq 65$ años. Luego de 9 semanas de seguimiento (con un rango que llega a 90 semanas), la tasa de abandono fue baja a moderada (23\%).

\section{Modelos de Pesario Utilizados}

= Gelhorn
= Anillo de membrana
= Cubo
= Anillo
= Donut
= Dish
= Anillo de incontinencia

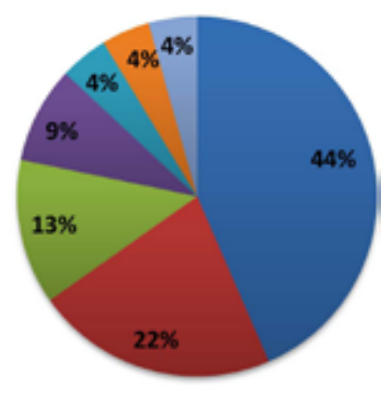

Figura 1. Frecuencia de modelos de pesarios utilizados. 


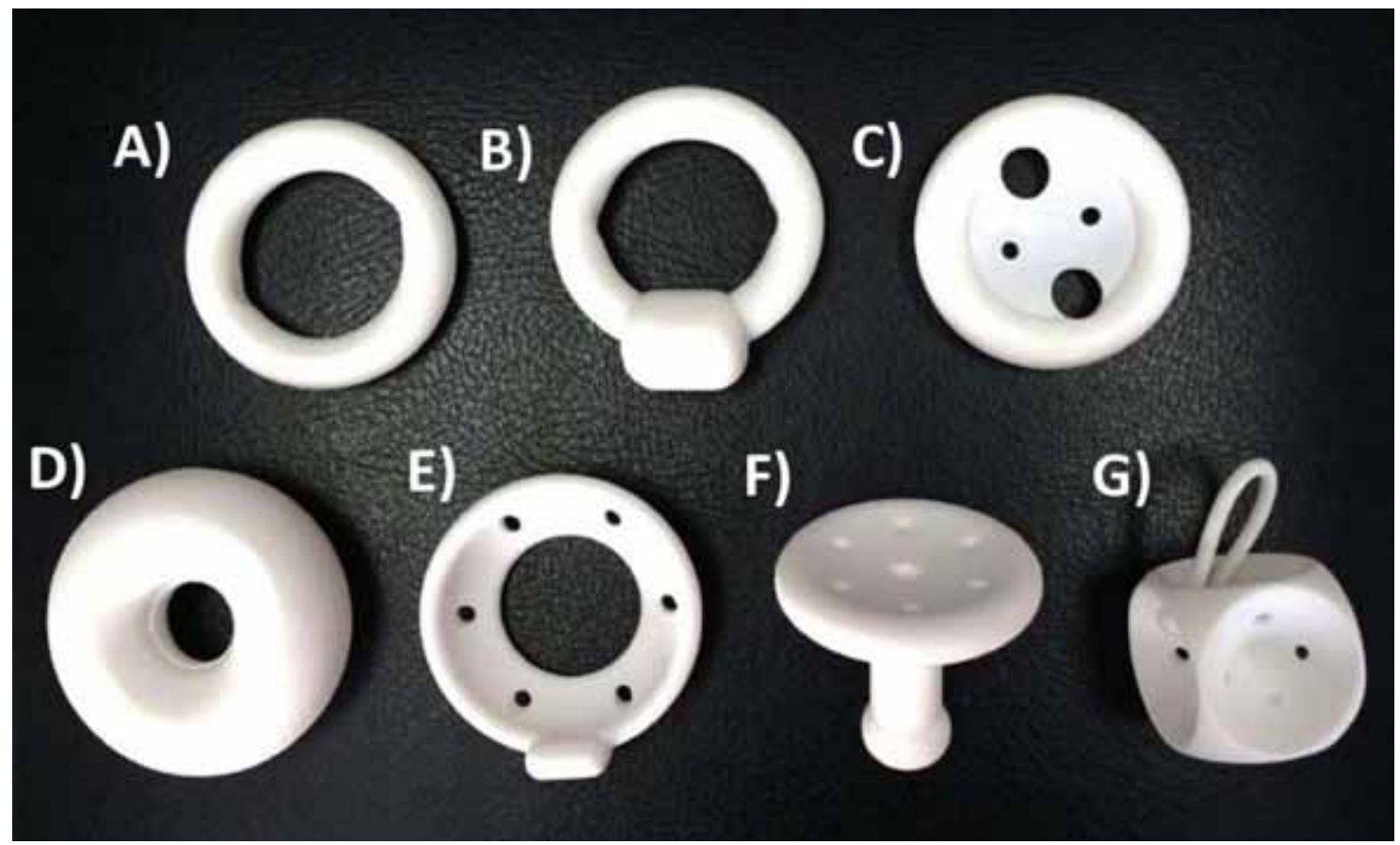

Figura 2. Modelos de pesarios utilizados en el estudio. A) Anillo B) Anillo de Incontinencia C) Anillo con membrana D) Donut E) Dish F) Gellhorn G) Cubo.

Tabla II

\section{EFECTOS ADVERSOS PRESENTADOS CON EL USO DE PESARIO EN PACIENTES $\leq 65$ AÑOS}

\begin{tabular}{lc} 
Efectos adversos & $\mathrm{n}$ \\
\hline Sensación de expulsión & 5 \\
Dolor & 5 \\
Flujo vaginal & 10 \\
Sangrado & 1 \\
Erosión & 3 \\
Incontinencia urinaria de esfuerzo & 3 \\
\hline
\end{tabular}

9 efectos adversos ocurrieron en 5 pacientes

De los resultados observados, los pesarios más tolerados y utilizados por las pacientes fueron el anillo con membrana y el Gellhorn. Esta preferencia es concordante con lo descrito en la literatura (6).

A pesar que un $52 \%$ de las pacientes refieren haber sufrido efectos adversos con el uso de pesarios, solo el $23 \%$ de los pacientes abandonaron el tratamiento. Todas estas complicaciones fueron manejadas sin dificultad, quedando libres de
Tabla III RESPUESTA SINTOMÁTICA SUBJETIVA
SEGÚN ESCALA PGI-I EN PACIENTES
$\leq 65$ AÑOS USUARIAS DE PESARIO

Respuesta sintomática

Mejoría subjetiva con PGI-I $64 \%(16 / 25)$

Pacientes que reportan estar $88 \%(14 / 16)^{\star}$ mejor

Mejoría en general (excelente, mucho mejor o un poco mejor)

Excelente

Mucho mejor

Un poco mejor

Sin cambio $1(10)$

* PGl disponible solo en 16 de las 25 pacientes

secuelas. Estas cifras son concordantes con los efectos adversos descritos en la literatura en usuarias de pesarios independiente de la edad (6).

Los hallazgos de este estudio son relevantes, especialmente en el contexto de hospitales con larga 
lista de espera para cirugía o de escasos recursos. El uso de pesarios es una alternativa mínimamente invasiva, de costo considerablemente menor que el tratamiento quirúrgico, con un aceptable perfil de seguridad y efectividad. Con los resultados obtenidos, parece plausible el uso de pesarios en pacientes $\leq 65$ años como opción de primera línea en la aproximación terapéutica del POP. De esta forma, el uso de pesarios podría no estar restringido a pacientes >65 años, a pesar de que hoy en día ésta sea la conducta más frecuente de los médicos.

\section{CONCLUSIÓN}

El uso de pesarios vaginales en pacientes menores de 65 años con POP es factible, asociado a mejoría subjetiva de los síntomas, con baja tasa de complicaciones. Son necesarios estudios prospectivos de mayor tamaño muestral para evaluar este subgrupo etario.

\section{REFERENCIAS}

1. Olsen A, Smith V, Bergstrom J. Epidemiology of surgically managed pelvicorgan prolapse and urinary incontinence. Obstet Gynecol 1997;89:501-6.

2. Digesu G A, Khullar V, Dudley C, Robinson L, Salvatore S.P-QOL: a validated questionnaire to assess the symptoms and quality of life of women with urogenital prolapse. Int Urogynecol J Pelvic Floor Dysfunct 2005;16:176-81.

3. Jelovsek J, Barber M. Women seeking treatment for advanced pelvic organ prolapse have decreased body image and quality of life. Am J Obstet Gynecol 2006;194:1455-61.

4. Flores EC, Araya GA, Pizarro-Berdichevsky J, Díaz RC, Quevedo CE, González LS. Descripción de la función sexual en mujeres con alteraciones de pisopélvico en un hospital público de Santiago. Rev Chil Obstet Ginecol 2012;77:331-7.
5. Atnip S, O'Dell K. Vaginal support pessaries: indications for use and fitting strategies. Urol Nurs 2012;32:114-24.

6. Lamers BH, Broekman BM, Milani AL. Pessary treatment for pelvic organprolapse and health-related quality of life: a review. Int Urogynecol J 2011;22:63744.

7. Mutone MF, Terry C, Hale DS, et al. Factors which influence the short-term success of pessary management of pelvic organ prolapse. Am J Obstet Gynecol 2005;193:89-94.

8. Powers K, Lazarou G, Wang A, et al. Pessary use in advanced pelvic organ prolapse. Int Urogynecol J 2006;17:160-4.

9. Bash K. Review of vaginal pessaries. Obstet Gynecol Surv 2000;55:455-60.

10. Kapoor DS, Thakar R, Sultan AH, Oliver R. Conservative versus surgical management of prolapse: what dictates patient choice? Int Urogynecol J Pelvic Floor Dysfunct 2009;20:1157-61.

11. Abdool Z, Thakar R, Sultan A H, Oliver RS. Prospective evaluation of outcome of vaginal pessaries versus surgery in women with symptomatic pelvic organ prolapsed. Int Urogynecol J 2011;22:273-8.

12. Clemons JL, Aguilar VC, Sokol ER, Jackson ND, Myers DL Patient characteristics that are associated with continued pessary use versus surgery after 1 year. Am J Obstet Gynecol 2004;19:159-64.

13. Atnip SD. Pessary use and management for pelvic organ prolapse. Obstet Gynecol Clin N Am 2009;36:54163.

14. Kuhn A, Bapst D, Standlmayr W, Vits K, Mueller MD. Sexual and organ function in patients with symptomatic prolapse: are pessaries helpful? Fertil Steril 2009;91:1914-8.

15. Bugge $C$, Hagen $S$, Thakar R. Vaginal pessaries for pelvic organ prolapse and urinary incontinence: a multiprofessional survey of practice. Int Urogynecol J 2013;24:1017-24.

16. Srikrishna S, Robinson D, Cardozo L. Validation of the Patient Global Impression of Improvement (PGI-I) for urogenital prolapse. Int Urogynecol J L 2010;21:523-8. 